\title{
COMPORTAMENTO DE GENÓTIPOS DE ABACAXIZEIRO RESISTENTES À FUSARIOSE EM COMPARAÇÃO A CULTIVARES COMERCIAIS SUSCETÍVEIS ${ }^{1}$
}

\author{
LUIZ CARLOS SANTOS CAETANO², JOSÉ AIRES VENTURA², \\ JOSÉ MAURO DE SOUSA BALBINO²
}

RESUMO - A fusariose é a doença mais severa na cultura do abacaxi no Brasil, e as cultivares mais plantadas, 'Pérola' e 'Smoth Cayenne', são suscetíveis, com perdas estimadas em 30 a 40\% dos frutos. O uso de genótipos resistentes à doença abre grande perspectiva para a solução do problema. O objetivo deste trabalho foi avaliar, em experimento de campo, o potencial produtivo, as características das plantas e a qualidade de frutos de novos híbridos de abacaxi resistentes à fusariose: EC-105, EC-93, cv. BRS Vitória e cv. BRS Imperial, comparando o desempenho destes genótipos com as cultivares 'Pérola', 'Smooth Cayenne' e 'Gold (MD-2)', suscetíveis à doença. O delineamento experimental foi o em blocos casualizados, com cinco repetições. A cv. BRS Imperial apresenta características importantes de qualidade de fruto, com maiores valores de SS e ratio. A cv. BRS Vitória reúne características favoráveis, como massa de frutos semelhante à da 'Pérola', reduzido diâmetro do eixo central do fruto, baixos índices de aderência de mudas "filhote" ao fruto e de tombamento de frutos, e boa produção de mudas aproveitáveis. Os demais híbridos resistentes à fusariose apresentam características negativas que limitam sua recomendação como cultivar comercial.

Termos para indexação: Ananas comosus, Fusarium guttiforme, cv. Vitória, cv. Imperial, características de plantas e de frutos.

\section{BEHAVIOR OF PINEAPPLE GENOTYPES FUSARIOSE RESISTANT COMPARED TO SUSCEPTIBLE COMMERCIAL CULTIVARS}

\begin{abstract}
The fusariose is the most severe disease in pineapple in Brazil and the most planted cultivars, 'Perola' and 'Smoth Cayenne', are susceptible, with estimated losses of 30 to $40 \%$ of the fruits. The use of disease resistant genotypes opens great prospects for resolving the problem. The objective of this work was to study, in field experiment, the potential of production, the plants characteristics and the fruit quality of new pineapple hybrids fusariosis resistant EC-105, EC-93, cv. BRS Vitória and cv. BRS Imperial comparing the performance of these genotypes with the cultivars 'Pérola', 'Smooth Cayenne' and 'Gold (MD-2)', susceptible to disease. The experimental design was completely randomized blocks, with five replicates. The cv. BRS Imperial features important fruit quality, with higher values of SS and ratio. The cv. BRS Vitoria brings together favorable characteristics as mass of fruit similar to the Pérola cultivar, reduced diameter of the central axis of the fruit, low rates of adherence of slips to fruit and fruit overturned, production of good number of profitable slips. The other hybrids resistant to fusariosis show negative characteristics that limit its recommendation to cultivate commercial.
\end{abstract}

Keywords: Ananas comosus, Fusarium guttiforme, cv. Vitória, cv. Imperial, characteristics of plants and fruits.

\footnotetext{
${ }^{1}$ (Trabalho 117-14). Recebido em: 28-03-2014. Aceito para publicação em: 09-10-2014.Trabalho realizado com recursos do CNPq. 2Engenheiro Agrônomo, D.Sc., Pesquisador do Incaper. E-mails: luizcaetano@incaper.es.gov.br; ventura@incaper.es.gov.br; balbino@incaper.es.gov.br
} 


\section{INTRODUÇÃO}

As cultivares de abacaxi mais plantadas no Brasil são Pérola e Smooth Cayenne (MATOS; REINHARDT, 2009). A cv. Gold (MD-2) desperta interesse pela possibilidade de exportação. Porém, esses genótipos são suscetíveis à fusariose, causada pelo fungo Fusarium guttiforme, doença mais severa na cultura do abacaxi no Brasil, com perdas estimadas de 30 a $40 \%$ dos frutos e até de $20 \%$ das mudas (VENTURA et al., 2009).

O controle da fusariose tem sido baseado na integração de práticas culturais e na aplicação de fungicidas quando do surgimento da inflorescência. $\mathrm{O}$ uso de variedade resistente, como 'BRS Imperial' (CABRAL; MATOS, 2009) e 'BRS Vitória' (VENTURA et al., 2009), certamente é mais econômico, eficiente e ambientalmente correto.

No Espírito Santo, o cultivo do abacaxizeiro é tradicional na região litorânea sul, com área plantada de cerca de 2.200 ha da cv. Pérola (IBGE, 2012). O controle químico da fusariose, além de caro e danoso ao homem e ao meio ambiente, temse mostrado pouco eficiente em virtude do plantio de mudas contaminadas e à não realização por parte dos produtores de práticas culturais como o roguing, para identificação e eliminação de plantas doentes.

A disponibilidade de genótipo resistente à doença, por si só, não garante as mudanças almejadas para a abacaxicultura, uma vez que a aceitabilidade comercial de um novo genótipo é fundamental para o sucesso do processo de substituição das variedades comerciais tradicionais, suscetíveis às doenças. Sendo assim, o objetivo deste trabalho foi avaliar o potencial produtivo, as características das plantas e a qualidade de frutos de genótipos de abacaxi resistentes à fusariose em comparação a cultivares comerciais suscetíveis.

\section{MATERIAL E MÉTODOS}

O experimento foi conduzido de maio de 2006 a junho de 2008, na Fazenda Experimental do Incaper de Pacotuba (20,75 S, 41,29 W e $130 \mathrm{~m})$, localizada no município de Cachoeiro de ItapemirimES. O clima da região é caracterizado como quente e úmido, precipitação média anual de $1.000 \mathrm{~mm}$, temperatura mínima do mês mais frio variando de 11,8 a $18,0^{\circ} \mathrm{C}$ e temperatura máxima do mês mais quente variando de 30,7 a $34,0^{\circ} \mathrm{C}$. Avaliaram-se sete genótipos de abacaxi: os híbridos EC-105, EC-93 e as cvs. BRS Vitória e BRS Imperial, resistentes à fusariose, e as cultivares comerciais Pérola, Gold (MD 2) e Smooth Cayenne, suscetíveis à fusariose.
Utilizaram-se no plantio mudas com cerca de $25 \mathrm{~cm}$ de altura provenientes do enviveiramento de mudas micropropagadas. As parcelas foram constituídas por três linhas duplas, no espaçamento de $0,90 \mathrm{x}$ $0,40 \times 0,30 \mathrm{~m}$, totalizando 72 plantas, avaliando-se dez plantas (centrais) da linha dupla do centro. O delineamento experimental utilizado foi o de blocos casualizados, com cinco repetições.

Os resultados das análises das amostras do solo (0 a $20 \mathrm{~cm}$ de profundidade) da área experimental foram: $\mathrm{pH}$ em água $=5,2 ; \mathrm{P}$ e K em $\mathrm{mg} \mathrm{dm}{ }^{-3}, 8,81$ e 99,0, respectivamente; $\mathrm{Ca}$ e $\mathrm{Mg}$, respectivamente, 2,88 e $0,87 \mathrm{cmol}_{\mathrm{c}} \mathrm{dm}^{-3} ; \mathrm{MO}=1,97$ dag $\mathrm{kg}^{-1}$; e micronutrientes (em $\mathrm{mg} \mathrm{dm}^{-3}$ ), $\mathrm{Zn}=6,8$; $\mathrm{Fe}=22,3 ; \mathrm{Mn}=88,8 ; \mathrm{Cu}=2,6$ e $\mathrm{B}=0,3$. A adubação do experimento foi realizada com as doses de 320 $\mathrm{kg} \mathrm{ha}^{-1} \mathrm{de} \mathrm{N}, 150 \mathrm{~kg} \mathrm{ha}^{-1}$ de $\mathrm{P}_{2} \mathrm{O}_{5}$ e de $320 \mathrm{~kg} \mathrm{ha}^{-1} \mathrm{de}$ $\mathrm{K}_{2} \mathrm{O}$. O fósforo foi aplicado parcelado, com $2 / 3$ no plantio e $1 / 3$ no segundo mês do plantio, enquanto o nitrogênio e o potássio foram parcelados em partes iguais no segundo, quinto e oitavo meses após o plantio. Foi feita uma aplicação foliar de ácido bórico a $0,3 \%$ antes da indução floral. A indução do florescimento foi feita aos 11 meses após o plantio com a aplicação de etephon na concentração de 500 ppm, mais ureia a $2 \%$.

Avaliaram-se as seguintes características: massa do fruto com e sem coroa; diâmetro do fruto medido na parte mediana; comprimento do fruto sem coroa; diâmetro do eixo central avaliado após corte transversal do fruto na parte mediana; sólidos solúveis (SS) medidos pelo ${ }^{\circ}$ Brix, acidez titulável (AT) do suco do fruto, determinando-se a relação SS/AT (ratio). As amostras para determinação de SS e AT foram retiradas de todos os frutos da área útil da parcela e foram constituídas de um disco de aproximadamente $1,0 \mathrm{~cm}$ de espessura da porção mediana de cada fruto. Avaliaram-se ainda o comprimento do pedúnculo medido no campo em cada planta útil com auxílio de uma régua graduada; a percentagem de plantas úteis com frutos tombados; a contagem no campo do número de mudas produzidas (tipo filhotes, filhotes rebentões e rebentões); a incidência de fendilhamento do eixo ou pedúnculo na base do fruto por ocasião da colheita; a epinescência e a aderência de mudas tipo filhote aos frutos. Esta última variável foi determinada por ocasião da colheita do fruto, contando-se o número de filhotes que ficaram presos ao fruto. Os dados obtidos foram submetidos à análise de variância, com comparação das médias pelo teste de Tukey, a $5 \%$ de probabilidade. 


\section{RESULTADOS E DISCUSSÃO}

O híbrido EC-93 apresentou a maior massa de frutos com coroa, $1.988 \mathrm{~g}$, enquanto a cv. Imperial, a menor, 926 g (Tabela 1). Segundo Cabral e Matos (2009), a cv. Imperial apresenta crescimento lento, característica que demanda adequação de época de plantio para obter-se um período mais longo de desenvolvimento da planta no campo até a indução floral, sob pena da produção de frutos menores. O híbrido EC-105 também se destacou com massa média de frutos de $1.751 \mathrm{~g}$. O mercado de fruta in natura remunera melhor os frutos grandes, mas os demais genótipos apresentaram bons resultados para esta variável, pois as massas de frutos foram maiores (Smooth Cayenne com 1.483 g e Gold (MD2) com $1.380 \mathrm{~g}$ ) ou não diferiram (BRS Vitória com $1.194 \mathrm{~g}$ ) da massa média do fruto da cv. Pérola (1.266 g), considerada como referência, por ser a mais plantada no Brasil. O abacaxi 'Pérola' produz frutos normalmente com massa de 1.000 a $1.500 \mathrm{~g}$ (REINHARDT et al., 2002), já na cv. BRS Vitória os frutos podem alcançar $1.500 \mathrm{~g}$ (VENTURA et al., 2009). Poder-se-ia esperar frutos maiores na cv. Smooth Cayenne que normalmente produz frutos com valor de massa próximo ou superior a 2.000 g, como obtidos por Teixeira et al. (2002). A massa média de frutos da cv. Gold (MD-2) neste trabalho pode ser considerada satisfatória, pois enquanto Neto et al. (2009), em média de cinco plantios realizados no Estado da Paraíba, obtiveram massa de frutos de 1.454 g, Guarçoni M. e Ventura (2011), em cultivo deste genótipo na região norte do Espírito Santo, produziram frutos com massa máxima de $1.277 \mathrm{~g}$. A massa de frutos sem coroa apresentou resposta semelhante à massa com coroa para os materiais avaliados.

O diâmetro de fruto do híbrido EC-93 foi superior, enquanto a cv. Imperial apresentou o menor diâmetro. As cvs. Smooth Cayenne e Gold (MD-2) e o híbrido EC-105 não diferiram entre si e apresentaram maior diâmetro em relação à 'Vitória' e 'Pérola' que foram estatisticamente iguais, com 10,6 e 10,9 cm, respectivamente. Segundo Ventura et al. (2009), o diâmetro médio do fruto do abacaxi 'Vitória' é de 12,0 cm em frutos de $1.500 \mathrm{~g}$ de massa. Frutos mais compridos foram produzidos pelos híbridos EC-93 e EC-105, e pela 'Pérola'. Os frutos de 'Smooth Cayenne' e de 'Vitória' não diferiram estatisticamente para esta variável. O comprimento dos frutos de 'Vitória' e de 'Gold MD-2' foi estatisticamente igual, enquanto 'Imperial' apresentou menor comprimento (Tabela 2). Independentemente do genótipo, a massa do fruto esteve fortemente correlacionada ao seu crescimento em diâmetro e comprimento (Tabela 3), o que vai ao encontro de resultado encontrado por Veloso et al. (2001) para a cv. Pérola.

O diâmetro do eixo central do fruto não deve ser grande para possibilitar maior quantidade de polpa comestível, constituindo uma característica desejável em uma cultivar (CABRAL, 1999). Na cv. Vitória, este valor foi de $1,0 \mathrm{~cm}$. Já o híbrido EC-93, com fruto de maior massa, apresentou o maior diâmetro do eixo, $2,8 \mathrm{~cm}$. Os demais genótipos apresentaram valores intermediários para esta característica (Tabela 1).

A cultivar Gold (MD-2) apresentou frutos com menor comprimento do pedúnculo, seguida das cultivares Smooth Cayenne, Imperial e Vitória. Os maiores pedúnculos foram medidos nos híbridos EC-93, EC-105 e, sobretudo, na cv. Pérola, o que concorda com a descrição de Reinhardt et al. (2002) para esta última cultivar. A variável porcentagem de plantas com frutos tombados teve comportamento semelhante ao da variável comprimento do pedúnculo, exceto para 'Smooth Cayenne', que apresentou elevada percentagem de frutos tombados, mesmo com comprimento de pedúnculo reduzido (Tabela 3). A análise de correlação linear entre estas duas variáveis mostrou correlação positiva, com $r$ $=0,70(\mathrm{p} \leq 0,01)$. Normalmente, em cultivares com pedúnculo longo, observa-se maior tombamento de frutos, sendo esta uma característica negativa, uma vez que frutos tombados são mais suscetíveis à queima solar e, portanto, demandando proteção (BELL et al., 2006), o que irá onerar o custo da produção.

A cultivar Imperial e o híbrido EC-93 apresentaram, respectivamente, $92,5 \%$ e $72,5 \%$ dos filhotes presos à base do fruto. Estes resultados foram obtidos mesmo tomando-se o cuidado especial na colheita, ou seja, fazendo-se a retirada dos frutos com uso de faca na tentativa de preservar as mudas. Os menores valores foram apresentados pelas cultivares Smooth Cayenne e Gold (MD-2), sem aderência das mudas filhotes aos frutos, mas, por outro lado, estas cultivares produziram as menores quantidades de mudas por planta, o que pode ter influenciado no resultado. As cultivares Vitória e Pérola não diferiram estatisticamente para esta característica (Tabela 3). Uma característica negativa em uma cultivar de abacaxi é a produção de mudas tipo filhote presas à base do fruto. Estas devem situar-se no mínimo a 2,0 cm de sua base (CABRAL, 1999), porque, do contrário, por ocasião da colheita do fruto, a muda solta-se da planta e, sendo muito pequena para plantio no campo, normalmente não é aproveitada. 
Acv. Pérola produziu o maior número médio de mudas tipo filhote, com 10,6 mudas por planta, seguida pela cultivar Imperial, com média de 9,0 filhotes por planta. As cultivares Smooth Cayenne e Gold (MD-2) apresentaram o menor número de filhotes por planta. O 'Vitória' produziu, em média, 6,6 filhotes por planta, maior do que o número de 4,2 descrito por Ventura et al. (2009) na caracterização da cultivar. Comportamento semelhante ocorreu com o número total de mudas (Tabela 3 ). O híbrido EC93 e a cultivar Imperial, apesar de produzirem boa quantidade de mudas, mostraram elevada aderência das mudas tipo filhote à base do fruto, o que leva à perda total ou parcial deste tipo de muda com a colheita do fruto. Entre os produtores, a produção de boa quantidade de mudas é uma característica desejável em uma cultivar de abacaxi, pois essas mudas serão usadas na formação de novas lavouras ou serão comercializadas.

Os maiores valores de SS foram encontrados nas cultivares Imperial e Smooth Cayenne. Exceto para o híbrido EC-105, os demais genótipos não diferiram da cultivar Pérola, que atualmente é a mais plantada. O maior valor de acidez titulável (AT) foi encontrado nos frutos da cv. Vitória, contudo sem diferir de 'Smooth Cayenne'. Este último, por sua vez, apresentou AT estatisticamente igual à cv. Gold (MD-2), que não diferiu dos demais genótipos. Os resultados de AT de 'Vitória' e 'Smooth Cayenne' refletiram em uma baixa relação $\mathrm{SS} / \mathrm{AT}$ (ratio) (Tabela 4). Segundo Ventura et al. (2009), a cv.Vitória apresenta AT superior e ratio inferior à cultivar Pérola. $\mathrm{O}$ maior valor de ratio foi obtido na cv. Imperial. Resultados semelhantes foram encontrados por Viana et al. (2013) analisando essas características nas cvs. BRS Vitória, BRS Imperial, Pérola, Smooth Cayenne e Gold, em trabalho de pesquisa conduzido no Estado da Bahia. O ratio é uma medida importante na avaliação do sabor do abacaxi, porém Berilli et al. (2011) encontraram que o teor de SS foi preponderante para os atributos sabor e impressão global em trabalho com as cvs. Vitória e Pérola, entre outras.

O híbrido EC-93 apresentou folhas com espinhos, que foram ausentes no EC-105 (Tabela 4). Verificou-se desuniformidade quanto à epinescência dentro do genótipo cv. Gold (MD-2), observando-se plantas com e sem espinhos nas folhas, demonstrando que o material plantado ainda precisa passar por um processo de seleção. Nas cvs. Imperial e Vitória, as folhas apresentaram-se desprovidas de espinhos, concordando com as descrições de Cabral e Matos (2009) e Ventura et al. (2009). A cv. Pérola apresentou espinhos na coroa do fruto e nas folhas, e na cv.
Smooth Cayenne estes estavam nas extremidades apicais, características que são inerentes a esses materiais (REINHARDT, 2002). A epinescência é um descritor morfológico relevante em programas de melhoramento genético do abacaxizeiro (CABRAL, 1999; VIEIRA et al., 2010), uma vez que a ausência de espinhos nas folhas é uma característica desejável em uma cultivar de abacaxizeiro, pois facilita os tratos culturais, sendo assim um fator importante na adoção por parte do produtor do plantio em fileiras duplas que promove a elevação do número de plantas por hectare, o que aliado ao plantio de um genótipo resistente à fusariose pode representar significativo acréscimo no número de frutos comerciais colhidos e, consequentemente, na renda do abacaxicultor.

Os híbridos EC-105 e EC-93 produziram frutos grandes, de massa elevada e com boas características de qualidade, mas caracteres negativos, como o tombamento e a alta aderência de filhotes à base do fruto, aliados à rachadura interna do eixo por ocasião da colheita $(36,8 \%$ dos frutos para EC105 e 38,2\% para EC-93), limitam estes genótipos para lançamento como cultivar comercial. Talvez a antecipação da colheita possa reduzir o problema da rachadura do eixo do fruto, mas estudos devem ser realizados para verificar a eficiência desta prática e sua influência na qualidade do fruto. Os demais genótipos não apresentaram o problema da rachadura interna do eixo. 
TABELA 1-Características dos frutos de sete genótipos de abacaxizeiro. Cachoeiro de Itapemirim, 2008.

\begin{tabular}{lccccc}
\hline Genótipo & $\begin{array}{c}\text { Massa com coroaMassa sem coroa Diâmetro do } \\
\text { (g) }\end{array}$ & $\begin{array}{c}\text { Comprimento } \\
(\mathbf{g})\end{array}$ & $\begin{array}{c}\text { Diâmetro do } \\
\text { fruto }(\mathbf{c m})\end{array}$ & $\begin{array}{c}\text { Sem coroa }(\mathbf{c m}) \\
\text { eixo central(cm) }\end{array}$ \\
\hline EC-93 & $1.988 \mathrm{a}$ & $1.894 \mathrm{a}$ & $12,6 \mathrm{a}$ & $19,7 \mathrm{a}$ & $2,8 \mathrm{a}$ \\
EC-105 & $1.751 \mathrm{~b}$ & $1.684 \mathrm{~b}$ & $11,8 \mathrm{~b}$ & $18,5 \mathrm{a}$ & $2,0 \mathrm{~b}$ \\
Smooth Cayenne & $1.483 \mathrm{c}$ & $1.311 \mathrm{c}$ & $11,4 \mathrm{bc}$ & $15,2 \mathrm{~b}$ & $2,0 \mathrm{~b}$ \\
Gold (MD-2) & $1.380 \mathrm{~cd}$ & $1.238 \mathrm{~cd}$ & $11,8 \mathrm{~b}$ & $13,6 \mathrm{c}$ & $2,1 \mathrm{~b}$ \\
Pérola & $1.266 \mathrm{de}$ & $1.175 \mathrm{~d}$ & $10,6 \mathrm{~d}$ & $18,8 \mathrm{a}$ & $1,6 \mathrm{c}$ \\
Vitória & $1.194 \mathrm{e}$ & $1.135 \mathrm{~d}$ & $10,9 \mathrm{~cd}$ & $14,7 \mathrm{bc}$ & $1,0 \mathrm{~d}$ \\
Imperial & $926 \mathrm{f}$ & $830 \mathrm{e}$ & $10,0 \mathrm{e}$ & $12,2 \mathrm{~d}$ & $1,7 \mathrm{c}$ \\
\hline CV (\%) & 4,9 & 4,9 & 2,0 & 3,8 & 4,2 \\
\hline
\end{tabular}

Médias seguidas pela mesma letra, nas colunas, não diferem ao nível de $5 \%$ de probabilidade, pelo teste de Tukey

TABELA 2- Matriz de correlação linear para massa média de frutos, com (PFCC) e sem coroa (PFSC), diâmetro de fruto (DF) e comprimento de fruto sem coroa (CF). Cachoeiro de Itapemirim, 2008.

\begin{tabular}{cccc}
\hline Variáveis & PFSC & DF & CF \\
\hline PFCC & $0,9914^{* *}$ & $0,9108^{* *}$ & $0,7440^{* *}$ \\
PFSC & - & $0,8876^{* *}$ & $0,7760^{* *}$ \\
DF & - & - & $0,5200^{* *}$ \\
\hline
\end{tabular}

*Significativo ao nível de $1 \%$ de probabilidade, pelo teste $\mathrm{t}$

TABELA 3- Comprimento do pedúnculo dos frutos, porcentagem de plantas com frutos tombados e com mudas tipo filhote aderidas ao fruto, número médio de mudas tipo filhote e número total ${ }^{1}$ de mudas por planta. Cachoeiro de Itapemirim, 2008

\begin{tabular}{lccccc}
\hline \multicolumn{1}{c}{ Genótipo } & $\begin{array}{c}\text { Pedúnculo } \\
(\mathrm{cm})\end{array}$ & $\begin{array}{c}\text { Tombamento } \\
\text { de frutos }(\%)\end{array}$ & $\begin{array}{c}\text { Aderência de filhotes } \\
\text { ao fruto }(\%)\end{array}$ & $\begin{array}{c}\text { № médio de } \\
\text { filhotes/planta }\end{array}$ & $\begin{array}{c}\text { № médio total } \\
\text { de mudas/planta }\end{array}$ \\
\hline EC-93 & $25,9 \mathrm{~b}$ & $47,5 \mathrm{a}$ & $72,5 \mathrm{ab}$ & $7,2 \mathrm{c}$ & $7,6 \mathrm{~b}$ \\
EC-105 & $24,6 \mathrm{~b}$ & $60,0 \mathrm{a}$ & $40,0 \mathrm{bc}$ & $3,8 \mathrm{~d}$ & $4,2 \mathrm{c}$ \\
S. Cayenne & $12,9 \mathrm{c}$ & $50,0 \mathrm{a}$ & $0,0 \mathrm{~d}$ & $0,8 \mathrm{e}$ & $2,8 \mathrm{c}$ \\
Gold (MD-2) & $8,3 \mathrm{~d}$ & $10,0 \mathrm{~b}$ & $0,0 \mathrm{~d}$ & $0,6 \mathrm{e}$ & $3,0 \mathrm{c}$ \\
Pérola & $28,4 \mathrm{a}$ & $52,5 \mathrm{a}$ & $22,5 \mathrm{~cd}$ & $10,6 \mathrm{a}$ & $10,8 \mathrm{a}$ \\
Vitória & $11,2 \mathrm{c}$ & $2,5 \mathrm{~b}$ & $37,5 \mathrm{c}$ & $6,6 \mathrm{c}$ & $8,0 \mathrm{~b}$ \\
Imperial & $12,9 \mathrm{c}$ & $12,5 \mathrm{~b}$ & $92,5 \mathrm{a}$ & $9,0 \mathrm{~b}$ & $11 \mathrm{a}$ \\
\hline CV $(\%)$ & 5,5 & 31,9 & 44,7 & 10,2 & 10,6 \\
\hline
\end{tabular}

Médias seguidas pela mesma letra, nas colunas, não diferem ao nível de $5 \%$ de probabilidade, pelo teste de Tukey. ${ }^{1}$ Total de mudas $=$ filhotes + filhote rebentão+rebentão

TABELA 4- Resultados de determinação de SS ( ${ }^{\circ}$ Brix), acidez titulável (AT), ratio e epinescência foliar em sete genótipos de abacaxizeiro. Cachoeiro de Itapemirim, 2008.

\begin{tabular}{lcccc}
\hline Genótipo & SS ( ${ }^{\circ}$ Brix) & AT (\%) & Ratio $^{\mathbf{1}}$ & Epinescência $^{\text {E }}$ \\
\hline EC-93 & $13,0 \mathrm{~b}$ & $0,28 \mathrm{c}$ & $46,4 \mathrm{~b}$ & Sim \\
EC-105 & $11,2 \mathrm{c}$ & $0,25 \mathrm{c}$ & $44,8 \mathrm{~b}$ & Não \\
S. Cayenne & $15,0 \mathrm{a}$ & $0,48 \mathrm{ab}$ & $31,2 \mathrm{bc}$ & Parcial \\
Gold (MD-2) & $14,7 \mathrm{ab}$ & $0,35 \mathrm{bc}$ & $42,0 \mathrm{~b}$ & Sim $_{\text {Não }}$ \\
Pérola & $13,1 \mathrm{~b}$ & $0,30 \mathrm{c}$ & $43,7 \mathrm{~b}$ & Sim \\
Vitória & $13,3 \mathrm{~b}$ & $0,53 \mathrm{a}$ & $25,1 \mathrm{c}$ & Não \\
Imperial & $15,8 \mathrm{a}$ & $0,28 \mathrm{c}$ & $56,4 \mathrm{a}$ & Não \\
\hline CV (\%) & 5,3 & 16,9 & 12,3 & \\
\hline
\end{tabular}

Médias seguidas pela mesma letra, nas colunas, não diferem ao nível de $5 \%$ de probabilidade, pelo teste de Tukey. ${ }^{1}$ Ratio=SS/AT. ${ }^{2}$ Observaram-se plantas com e sem espinhos nas parcelas. 


\section{CONCLUSÕES}

A cv. Imperial apresenta características importantes de qualidade de fruto, com maiores valores de SS e ratio.

A cv.Vitória reúne características favoráveis, como massa de frutos semelhante ao 'Pérola', reduzido diâmetro do eixo central do fruto, baixos índices de aderência de mudas "filhote" ao fruto e de tombamento de frutos, e boa produção de mudas aproveitáveis.

Os demais híbridos resistentes à fusariose apresentam características negativas que limitam a sua recomendação para plantio comercial.

\section{REFERÊNCIAS}

BELL, D.; ORTIZ, V.R.; SCOTT, C.; PHILLIPS, N. Surround crop protectant - for the reduction of sunburn damage and heat stress in pineapple. Acta Horticulturae, The Hague, v.702, p. 179-184, 2006.

BERILLI, S.S.; ALMEIDA, S.B.; CARVALHO, A.J.C.; FREITAS, S.J.; BERILLI, A.P.C.G.; SANTOS, P.C. Avaliação sensorial dos frutos de cultivares de abacaxi para consumo in natura. Revista Brasileira de Fruticultura, Jaboticabal, p.592-598, 2011. Volume Especial.

CABRAL, J.R.S. Melhoramento genético. In: CUNHA, G.A.; CABRAL, J.R.S.; SOUZA, L.F.S. O abacaxizeiro: cultivo, agroindústria e economia. Brasília: Embrapa, 1999. p.83-104.

CABRAL, J.R.S.; MATOS, A.P. Imperial, a new pineapple cultivar resistant to fusariose. Acta Horticulturae, The Hague, v. 822, p. 47-50, 2009.

GUARÇONI, M. A.; VENTURA, J.A. Adubação N-P-K e o desenvolvimento, produtividade e qualidade dos frutos do abacaxi 'Gold' (MD-2). Revista Brasileira de Ciência do Solo, Viçosa, MG, v.35, p.1.367-1.376, 2011.

IBGE. SIDRA: Sistema de recuperação automática. 2012. Disponível em: http://www.ibge/sidra.org.br. Acesso em: 12 ago. 2014.
MATOS, A.P.; REINHARDT, D.H.R.C. Pineapple in Brazil: Characteristics, research and perspectives. Acta Horticulturae, The Hague, v. 822, p. 25-36, 2009.

NETO, M.B.; LACERDA, J.T.; CARVALHO, R.A.; OLIVEIRA, E.F. Comportamento do abacaxixeiro 'MD-2' na Paraíba. Tecnologia \& Ciência Agropecuária, João Pessoa, v.3, n.3, p.19-22, 2009.

REINHARDT, D.H.; CABRAL, J.R.C.; SOUZA, L.F.S.; SANCHES, N.F.; MATOS, A.P. Pérola and Smooth Cayenne pineapple cultivars in the state of Bahia, Brazil: growth, flowering, pests, diseases, yield and fruit quality aspects. Fruits, Paris, v. 57, p. 43-53, 2002.

TEIXEIRA, L.A.J.; SPIRONELLO,A.; FURLANI, P.R.;SIGRIST, J.M.M. Parcelamento da adubação NPK em abacaxizeiro. Revista Brasileira de Fruticultura, Jaboticabal, v.24, n.1, p.219-224, 2002.

VELOSO, C.A.C.; OEIRAS, A.H.L.; CARVALHO, E.J.M.; SOUZA, F.R. Resposta do abacaxizeiro à adição de nitrogênio, potássio e calcário em Latossolo Amarelo do nordeste paraense. Revista Brasileira de Fruticultura, Jaboticabal, v.23, n.2, p.396-402, 2001.

VENTURA, J.A.; CABRAL, J.R.S.;MATOS, A.P. 'Vitória': new pineapple cultivar resistant to fusariosis. Acta Horticulturae, The Hague, v. 822, p.51-55, 2009.

VIANA, E.S.; REIS, R.C.;JESUS, J.L.; JUNGHANS, D.T.; SOUZA, F.V.D. Caracterização físico-química de novos híbridos de abacaxi resistentes à fusariose. Ciência Rural, Santa Maria, v.43, n.7, p.1155-1161, 2013.

VIEIRA, L.J.; SANTOS, L.R.; CASTELEN, M.S.; JUNGHANS, D.T. Caracterização morfológica de acessos de abacaxizeiro. C\&D- Revista Eletrônica da Fainor, Vitória da Conquista, v.3, n.1, p. 68-77, 2010 . 